\title{
THRESHOLD BEHAVIOUR OF EMERGING EPIDEMICS FEATURING CONTACT TRACING
}

\author{
FRANK G. BALL, $* * *$ \\ EDWARD S. KNOCK $* * * *$ AND \\ PHILIP D. O'NEILL, ${ }^{* * * * *}$ University of Nottingham
}

\begin{abstract}
This paper is concerned with a stochastic model for the spread of an epidemic with a contact tracing scheme, in which diagnosed individuals may name some of their infectious contacts, who are then removed if they have not been already. Traced individuals may or may not also be asked to name their own contacts. The epidemic is studied by considering an approximating, modified birth-death process with intersibling dependencies, for which a threshold parameter and expressions from which extinction probabilities may be calculated are derived. When all individuals can name their contacts, it is shown that this threshold parameter depends on the infectious period distribution only through its mean. Numerical studies show that the infectious period distribution choice can have a material effect on the threshold behaviour of an epidemic, while the dependencies help reduce spread.
\end{abstract}

Keywords: Stochastic epidemic; contact tracing; branching process; reproduction number

2010 Mathematics Subject Classification: Primary 92D30

Secondary $60 \mathrm{~J} 80$

\section{Introduction}

This paper is concerned with a stochastic model for the spread of an infectious disease amongst a homogeneously mixing population. The model features a contact tracing scheme in which individuals can name their infectious contacts upon diagnosis, with traced contacts being prevented from making further potentially infectious contacts. Unlike previous stochastic models for contact tracing, such as Müller et al. (2000) and Klinkenberg et al. (2006), we do not assume that all infected individuals will eventually be asked to name contacts, since we assume that there is a fixed probability that a traced individual will be asked to name their contacts. Other tracing models may involve tracing neighbours of diagnosed individuals in a networkstructured population (see, e.g. Shaban et al. (2008)) or housemates of diagnosed individuals in a household-structured population (see, e.g. Ball et al. (2007) and Ball et al. (2008)); the tracing in this paper differs from these in that traced individuals are necessarily infected. The tracing differs from a traced vaccination model, such as that of Kaplan et al. (2002) (in which traced individuals are vaccinated but may still make further contacts), in that we assume that traced individuals cannot make further contacts. Furthermore, the model of Kaplan et al. is an

Received 18 January 2011; revision received 19 July 2011.

* Postal address: School of Mathematical Sciences, The University of Nottingham, University Park, Nottingham, NG7 2RD, UK.

** Email address: frank.ball@ @ottingham.ac.uk

*** Email address: edwardknock@gmail.com

**** Email address: philip.oneill@ nottingham.ac.uk 
example of a deterministic contact tracing model; by using a stochastic environment instead we are able to determine (in some cases) how likely the tracing is to be effective in reducing spread.

In this paper, the threshold behaviour of the epidemic process is studied via a related modified birth-death process that is a novel example of a branching process in continuous-time with dependencies, in that the lifetimes of siblings are codependent on their parent's lifetime. Types of dependencies explored previously in discrete-time branching processes include: populationsize-dependent branching processes (see, e.g. Klebaner (1989)), sibling-dependent branching processes (see, e.g. Olofsson (1996)), and branching processes in varying environments (see, e.g. Athreya and Karlin (1971)).

By and large the dependencies in epidemic models with contact tracing make the analysis of related branching processes difficult. Previous studies of contact tracing in a stochastic environment have used such analytical methods as truncation of naming chains (see, e.g. Klinkenberg et al. (2006)), bounding approximations (see, e.g. Becker et al. (2005)), and asymptotic threshold parameters (see, e.g. Müller et al. (2000)). In this paper we use an analytical framework that not only allows us to obtain exact expressions for a threshold parameter, but also, in some situations, expressions for calculating extinction probabilities. Furthermore, we compare these results with some obtained for approximations which assume that sibling units are independent, in order to examine the effect of the dependencies. Our model does not allow for susceptible individuals to be traced, but some of the theory in this paper can be extended to a model which incorporates a latent period and a delay in tracing, the analysis of which is more complicated and will be presented in a separate paper.

The paper is structured as follows. The epidemic with contact tracing model is defined in Section 2. A modified birth-death process is also defined and its use to determine the threshold behaviour of the epidemic process is outlined. Threshold parameters and expressions for extinction probabilities are derived for constant and exponentially distributed infectious periods in Sections 3 and 4, respectively. In Section 5 it is shown that in the iterative tracing case the threshold parameter depends only on the infectious period distribution through its mean. Section 6 contains some numerical illustrations of the theory.

\section{Model and threshold behaviour}

\subsection{Epidemic and contact tracing models}

We consider an SIR (susceptible $\rightarrow$ infective $\rightarrow$ removed) epidemic spreading amongst a homogeneously mixing closed population of size $N$, with a contract tracing scheme applied to reduce spread. At any time, each individual in the population is in one of three states: susceptible, infective, or removed. Initially, a small number of individuals are infective and the rest are susceptible. Contacts between any two given individuals in the population occur at times given by the points of a homogeneous Poisson process with rate $\lambda / N$. A susceptible individual becomes an infective individual if he/she makes contact with an infective. An infective individual remains infectious for a period of time distributed according to a random variable $T_{I}$, having an arbitrary but specified distribution, and then becomes removed. Once removed, an individual no longer plays a part in the epidemic process. The epidemic ends when there is no infective individual left in the population. All the Poisson processes and random variables describing infectious periods are assumed to be mutually independent.

A contact tracing scheme is incorporated as follows. Upon removal, an individual (i.e. an infector) names each of the individuals they infected (i.e. their infectees), if any, independently 
with probability $p$, and named infectees are removed immediately. An individual whose removal is a result of contact tracing (and not the natural end of their infectious period) is said to be traced. Note that a named individual is not traced if their infectious period ends during their infector's infectious period (at which time they will of course be asked to name their own infectees). With probability $\pi$, a traced individual names their own infectees in the same manner (i.e. independently with probability $p$ ) with named infectees being removed (this occurs immediately when the traced individual is removed), otherwise they name none of their infectees. We adopt the convention of describing an individual as named throughout their life, as long as they will be named during the epidemic. The naming process is assumed independent of the Poisson processes and random variables describing infectious periods. Special cases of particular interest are when $\pi=0$ (no traced individual is asked to name their contacts) and when $\pi=1$ (all individuals are asked to name their contacts), which we refer to as single-step and iterative tracing, respectively.

\subsection{Threshold behaviour and a modified birth-death process}

If the population size $N$ is large then, during the early stages of the epidemic, there is only a small probability that an infective makes contact with an already infected individual. Thus, we can approximate the early stages of the epidemic by a process in which all an infective's contacts are made with susceptible individuals. In this approximation, the process of infected individuals follows a modified birth-death process. The approximation can be made fully rigorous by considering a sequence of epidemics, indexed by $N$, and modifying the coupling argument of Ball (1983) (see also Ball and Donnelly (1995)) to prove almost-sure convergence of the epidemic model with tracing to the modified birth-death process as $N \rightarrow \infty$.

This modified birth-death process, with births corresponding to new infections and deaths corresponding to removals, is as follows. Individuals give birth at rate $\lambda$ over their lifetime which has a natural length distributed as $T_{I}$. Furthermore, when an individual dies naturally (i.e. without being traced), each of its offspring is named, independently and with probability $p$. An individual who is still alive at the time they are named dies unnaturally. When an individual dies unnaturally then, with probability $\pi$, they name their offspring in the same manner (again with named offspring dying unnaturally if they are alive when named), otherwise all their offspring will die naturally.

The threshold behaviour of this modified birth-death process can be obtained by considering the embedded single-type discrete-time Galton-Watson process describing unnamed individuals, in which the offspring of a given individual are either (a) their immediate unnamed offspring, or (b) unnamed descendants who are separated from the given individual, in the family tree, only by named individuals. To distinguish between the processes and for conciseness, we refer to the modified birth-death process as the MBDP and the Galton-Watson process as the GWP.

Let a named individual who is separated from its most recent unnamed ancestor in the family tree of the MBDP by $k-1$ named individuals be called a type- $k$ individual. Hence, type- 1 individuals are the named immediate offspring of unnamed individuals, type- 2 individuals are the named immediate offspring of the named immediate offspring of unnamed individuals, and so on. Type-0 individuals are unnamed individuals.

To analyse the MBDP, we focus attention on the offspring random variable, $R$ say, in the GWP, by obtaining expressions for its mean, $R_{U}=\mathrm{E}[R]$, which we call a type-reproduction number, following Heesterbeek and Roberts (2007). For some example realizations of $R$, see Figure 1 (the quantities $R_{k}$ (for $k=1,2, \ldots$ ) and $R^{(k)}$ (for $k=0,1, \ldots$ ) are defined in Section 4). Standard results from branching process theory tell us that the GWP dies out with 
(i)

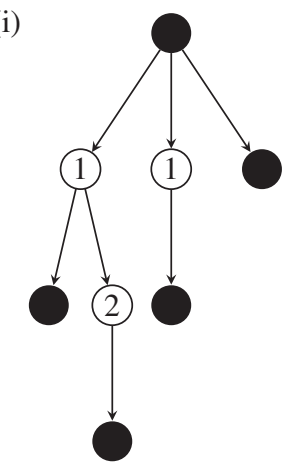

(ii)

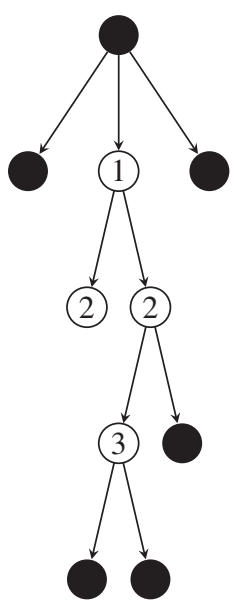

(iii)

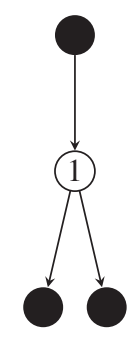

$=$ Type-0 (unnamed) individual
$k=$ Type $k$ (named) individual

FIGURE 1: Some example realizations of $R$. In (i) $R=4, R_{1}=2, R_{2}=1, R^{(0)}=1, R^{(1)}=3$, and $R^{(2)}=4$. In (ii) $R=5, R_{1}=0, R_{2}=1, R_{3}=2, R^{(0)}=2, R^{(1)}=2, R^{(2)}=3$, and $R^{(3)}=5$. In (iii) $R=2, R_{1}=2, R^{(0)}=0$, and $R^{(1)}=2$.

probability 1 if and only if $R_{U} \leq 1$, and that if $R_{U}>1$ and there is a single ancestor, who is unnamed, then the extinction probability of the GWP, $p_{\mathrm{e}}$ say, is the smallest solution of $s=H(s)$ in $(0,1)$, where $H(s)=\mathrm{E}\left[s^{R}\right]$. Note that the MBDP goes extinct if and only if the GWP goes extinct, and so the extinction probability of the MBDP equals that of the GWP.

\section{Constant infectious period}

In this section we assume that $T_{I} \equiv \iota$, and obtain expressions for $R_{U}$ and $H(s)$ by analysing the tree length of a two-type pure-birth process over $[0, \iota]$. We use a method of looking back along the tree which is similar to, but different from, that used in Lambert (2010).

Consider a typical unnamed individual, $A$ say, in the MBDP who has a lifetime of length $\iota$. It follows from the definition of the MBDP that each individual gives birth to unnamed individuals over their lifetime at the points of a Poisson process (independently of all other individuals) with rate $\lambda(1-p)$ if they are asked to name their offspring or rate $\lambda$ if they are not. Letting $D$ be the length of $A$ 's family tree of named individuals in the MBDP (i.e. the sum of the lifetimes of $A$ and all named descendants of $A$ who are separated from $A$ only by named individuals), we can write $D=D_{1}+D_{2}$, where $D_{1}$ and $D_{2}$ are the total lifetimes of individuals who can and cannot name offspring, respectively. The number of offspring $A$ has in the GWP therefore has a Poisson distribution with random mean $\lambda(1-p) D_{1}+\lambda D_{2}$, so $R_{U}=\mathrm{E}\left[\lambda(1-p) D_{1}+\lambda D_{2}\right]$ and $H(s)=$ $\psi(\lambda(1-p)(1-s), \lambda(1-s)), 0 \leq s \leq 1$, where $\psi\left(\theta_{1}, \theta_{2}\right)=\mathrm{E}\left[\mathrm{e}^{-\theta_{1} D_{1}-\theta_{2} D_{2}}\right], \theta_{1}, \theta_{2} \geq 0$. Furthermore, since all the individuals (other than $A$ ) who contribute to $D$ live until the end of $A$ 's lifetime and then die unnaturally, $D$ is equal in distribution to the tree length at time $\iota$ of a two-type (potent and impotent individuals) pure-birth process with one potent initial individual, in which potent individuals give birth to potent and impotent individuals at rates $\lambda p \pi$ and $\lambda p(1-\pi)$, respectively, over $[0, \iota]$ and impotent individuals have no offspring. Also, $D_{1}$ is equal in distribution to the tree length at time $\iota$ of the embedded single-type pure-birth process of potent individuals (which henceforth we refer to as the PBP). 
Consider the initial individual in the PBP, $\hat{A}$, say. We analyse $D$ by conditioning back from time $\iota$ to $\hat{A}$ 's last birth. Let the time of this birth be $\iota-\tilde{T}$, with $\tilde{T}=\iota$ if $\hat{A}$ has no offspring. Then $\tilde{T}$ has a probability mass of $\mathrm{e}^{-\lambda p \pi \iota}$ at $\iota$, while, if $\tilde{T}<\iota$ (which happens with probability $\left.1-\mathrm{e}^{-\lambda p \pi \iota}\right), \tilde{T}$ has an $\operatorname{Exp}(\lambda \pi p)$ distribution restricted to $[0, \iota)$, where the notation $\operatorname{Exp}(\mu)$ denotes an exponential distribution with mean $\mu^{-1}$. If $\tilde{T}=\iota$ (i.e. $\hat{A}$ has no births) then clearly $D_{1}=\iota$. If $\tilde{T}=t(t<\iota)$ then we know that over $[\iota-t, \iota]$ (i.e. a part of the tree of length $t$ ), $\hat{A}$ has no births. The remainder of the tree length is determined by considering the first $\iota-t$ time units of $\hat{A}$ 's lifetime, and the $t$ time units of the offspring's lifetime (which begins at time $\iota-t)$. These two time intervals are equivalent to the lifetime $\iota$ of a single individual. Thus, the remaining tree length is independent of and identically distributed to $D_{1}$, and it can be analysed by conditioning back again from time $\iota$.

Each time we condition back a length of time $t$ to a birth, there may be associated impotent births during this time in the two-type process. This number of births has a Poisson distribution with mean $\lambda p(1-\pi) t$ (which we denote by $\operatorname{Po}(\lambda p(1-\pi) t)$ ), and given this number, the lifetimes of the resulting offspring can be sampled from independent $U(0, t)$ distributions. Let the associated total length of impotent lifetimes when conditioning back a time $t$ be $Y_{t}$, and let $\phi_{t}(\theta)=\mathrm{E}\left[\mathrm{e}^{-\theta Y_{t}}\right], \theta \geq 0$. Thus, $\mathrm{E}\left[Y_{t}\right]=\lambda p(1-\pi) t^{2} / 2$ while, for $\theta \geq 0$,

$$
\begin{aligned}
\phi_{t}(\theta) & =\sum_{k=0}^{\infty} \mathrm{e}^{-\lambda p(1-\pi) t} \frac{(\lambda p(1-\pi) t)^{k}}{k !}\left(\int_{0}^{t} \frac{1}{t} \mathrm{e}^{-\theta u} \mathrm{~d} u\right)^{k} \\
& =\mathrm{e}^{-\lambda p(1-\pi) t} \exp \left\{\frac{\lambda p(1-\pi)}{\theta}\left(1-\mathrm{e}^{-\theta t}\right)\right\} .
\end{aligned}
$$

For $\omega_{1}, \omega_{2} \geq 0$, let $f\left(\omega_{1}, \omega_{2}\right)=\mathrm{E}\left[\omega_{1} D_{1}+\omega_{2} D_{2}\right]$. Then, from the above,

$$
\mathrm{E}\left[\omega_{1} D_{1}+\omega_{2} D_{2} \mid \tilde{T}=t\right]= \begin{cases}\omega_{1} \iota+\omega_{2} \frac{\lambda p(1-\pi) \iota^{2}}{2} & \text { if } t=\iota, \\ f\left(\omega_{1}, \omega_{2}\right)+\omega_{1} t+\omega_{2} \frac{\lambda p(1-\pi) t^{2}}{2} & \text { if } t<\iota,\end{cases}
$$

while, for $\left(\theta_{1}, \theta_{2}\right) \in \mathbb{R}_{+}^{2}$,

$$
\mathrm{E}\left[\mathrm{e}^{-\theta_{1} D_{1}-\theta_{2} D_{2}} \mid \tilde{T}=t\right]= \begin{cases}\mathrm{e}^{-\theta_{1} \iota} \phi_{\iota}(\theta) & \text { if } t=\iota, \\ \psi\left(\theta_{1}, \theta_{2}\right) \mathrm{e}^{-\theta_{1} t} \phi_{t}(\theta) & \text { if } t<\iota .\end{cases}
$$

Thus, letting $f_{\tilde{T} \mid \tilde{T}<\iota}$ be the probability density function of $\tilde{T}$ conditional upon $\tilde{T}<\iota$,

$$
\begin{aligned}
f\left(\omega_{1}, \omega_{2}\right)= & \mathrm{P}(\tilde{T}=\iota) \mathrm{E}\left[\omega_{1} D_{1}+\omega_{2} D_{2} \mid \tilde{T}=\iota\right] \\
& +\mathrm{P}(\tilde{T}<\iota) \int_{0}^{\iota} f_{\tilde{T} \mid \tilde{T}<\iota}(t) \mathrm{E}\left[\omega_{1} D_{1}+\omega_{2} D_{2} \mid \tilde{T}=t\right] \mathrm{d} t \\
= & \frac{\omega_{1}}{\lambda p \pi}\left(1-\mathrm{e}^{-\lambda p \pi \iota}\right)+\left(1-\mathrm{e}^{-\lambda p \pi \iota}\right) f\left(\omega_{1}, \omega_{2}\right) \\
& +\frac{\omega_{2}(1-\pi)}{\pi}\left(\frac{1}{\lambda p \pi}-\frac{1}{\lambda p \pi} \mathrm{e}^{-\lambda p \pi \iota}-\iota \mathrm{e}^{-\lambda p \pi \iota}\right),
\end{aligned}
$$

which on rearranging gives

$$
f\left(\omega_{1}, \omega_{2}\right)=\frac{\omega_{1}}{\lambda p \pi}\left(\mathrm{e}^{\lambda p \pi \iota}-1\right)+\frac{\omega_{2}(1-\pi)}{\pi}\left(\frac{1}{\lambda p \pi} \mathrm{e}^{\lambda p \pi \iota}-\frac{1}{\lambda p \pi}-\iota\right) .
$$


Setting $\omega_{1}=\lambda(1-p)$ and $\omega_{2}=\lambda$ in the above yields

$$
R_{U}=\frac{1-p}{p \pi}\left(\mathrm{e}^{\lambda p \pi \iota}-1\right)+\frac{1-\pi}{\pi}\left(\frac{1}{p \pi} \mathrm{e}^{\lambda p \pi \iota}-\frac{1}{p \pi}-\lambda \iota\right) .
$$

Meanwhile, for $\theta_{1}, \theta_{2}>0$,

$$
\begin{aligned}
\psi\left(\theta_{1}, \theta_{2}\right)= & \mathrm{P}(\tilde{T}=\iota) \mathrm{E}\left[\mathrm{e}^{-\theta_{1} D_{1}-\theta_{2} D_{2}} \mid \tilde{T}=\iota\right] \\
& +\mathrm{P}(\tilde{T}<\iota) \int_{0}^{\iota} f_{\tilde{T} \mid \tilde{T}<\iota}(t) \mathrm{E}\left[\mathrm{e}^{-\theta_{1} D_{1}-\theta_{2} D_{2}} \mid \tilde{T}=t\right] \mathrm{d} t \\
= & \mathrm{e}^{-\lambda p \pi \iota} \mathrm{e}^{-\theta_{1} \iota} \phi_{\iota}\left(\theta_{2}\right)+\left(1-\mathrm{e}^{-\lambda p \pi \iota}\right) \int_{0}^{\iota} \frac{\lambda p \pi \mathrm{e}^{-\lambda p \pi t}}{1-\mathrm{e}^{-\lambda p \pi \iota} \psi\left(\theta_{1}, \theta_{2}\right) \mathrm{e}^{-\theta_{1} t} \phi_{t}\left(\theta_{2}\right) \mathrm{d} t} \\
= & \mathrm{e}^{-\left(\lambda p+\theta_{1}\right) \iota} \exp \left\{\frac{\lambda p(1-\pi)}{\theta_{2}}\left(1-\mathrm{e}^{-\theta_{2} \iota}\right)\right\} \\
& +\psi\left(\theta_{1}, \theta_{2}\right) \lambda p \pi \mathrm{e}^{\lambda p(1-\pi) / \theta_{2}} \sum_{k=0}^{\infty} \frac{\left(-\lambda p(1-\pi) / \theta_{2}\right)^{k}}{k !\left(\lambda p+\theta_{1}+k \theta_{2}\right)}\left(1-\mathrm{e}^{-\left(\lambda p+\theta_{1}+k \theta_{2}\right) \iota}\right),
\end{aligned}
$$

whence

$$
\begin{aligned}
\psi\left(\theta_{1}, \theta_{2}\right)= & \mathrm{e}^{-\left(\lambda p+\theta_{1}\right) \iota} \exp \left\{\frac{\lambda p(1-\pi)}{\theta_{2}}\left(1-\mathrm{e}^{-\theta_{2} \iota}\right)\right\} \\
& \times\left(1-\lambda p \pi \mathrm{e}^{\lambda p(1-\pi) / \theta_{2}} \sum_{k=0}^{\infty} \frac{\left(-\lambda p(1-\pi) / \theta_{2}\right)^{k}}{k !\left(\lambda p+\theta_{1}+k \theta_{2}\right)}\left(1-\mathrm{e}^{-\left(\lambda p+\theta_{1}+k \theta_{2}\right) \iota}\right)\right)^{-1}
\end{aligned}
$$

and, for $0 \leq s<1$,

$$
\begin{aligned}
& H(s)= \mathrm{e}^{-(\lambda(1-(1-p) s)) \iota} \exp \left\{\frac{p(1-\pi)}{1-s}\left(1-\mathrm{e}^{-\lambda(1-s) \iota}\right)\right\} \\
& \times\left(1-\lambda p \pi \mathrm{e}^{p(1-\pi) /(1-s)} \sum_{k=0}^{\infty}\left\{\frac{(-p(1-\pi) /(1-s))^{k}}{k ! \lambda((k+1)(1-s)+p s)}\right.\right. \\
&\left.\left.\times\left(1-\mathrm{e}^{-\lambda((k+1)(1-s)+p s) \iota}\right)\right\}\right)^{-1} .
\end{aligned}
$$

\section{Exponential infectious period}

We obtain $R_{U}$ by considering a typical unnamed individual, $A$ say, in the MBDP. Let $R_{i}$ be the total number of unnamed descendants of $A$ in the MBDP who have exactly $i$ named individuals and no unnamed individual between themselves and $A$ in the family tree, and let $R_{U, i}=\mathrm{E}\left[R_{i}\right]$. Let $R^{(k)}$ be the total number of unnamed descendants of $A$ in the MBDP who have no unnamed individual and at most $k$ named individuals between themselves and $A$ in the family tree. Then, for $k=1,2, \ldots$,

$$
R^{(k)}=R^{(0)}+\sum_{i=1}^{k} R_{i}
$$


noting that $R^{(0)}$ is the number of unnamed immediate offspring of $A$. Examples of $R_{i}$ and $R^{(k)}$ are given in Figure 1. Let $R_{U}^{(k)}=\mathrm{E}\left[R^{(k)}\right]$. We have $R^{(k)} \uparrow R$ as $k \rightarrow \infty$, so, by the monotone convergence theorem,

$$
R_{U}=\lim _{k \rightarrow \infty} R_{U}^{(k)}
$$

Let $T$ denote the lifetime of our typical unnamed individual, $A$, in the $\operatorname{MBDP}(T \sim \operatorname{Exp}(\gamma))$ and, for $k=0,1, \ldots$, let $h_{k}(t)=\mathrm{E}\left[R^{(k)} \mid T=t\right]$. Now, $R^{(0)}$ is just the number of unnamed immediate offspring of $A$, so $\left(R^{(0)} \mid T=t\right) \sim \operatorname{Po}(\lambda(1-p) t)$, and $h_{0}(t)=\lambda(1-p) t$.

Let $N_{1}$ denote the number of named immediate (i.e. type-1) offspring of our typical individual and, for $i=1,2, \ldots, N_{1}$, let $Z_{i}^{(k)}$ be the total number of descendants from the $i$ th such (arbitrarily ordered) individual that contribute to $R^{(k)}$. Thus,

$$
\sum_{i=1}^{k} R_{i}=\sum_{i=1}^{N_{1}} Z_{i}^{(k)}
$$

In this paper all sums are 0 if vacuous. Now $\left(N_{1} \mid T=t\right) \sim \operatorname{Po}(\lambda p t)$ and, conditional upon $N_{1}=n$ and $T=t$, the birth times of these $n$ individuals can be obtained by sampling $n$ independent $U(0, t)$ random variables, so the $Z_{i}^{(k)}$ are independent and identically distributed, with common distribution $Z^{(k)}$, say. Thus,

$$
\mathrm{E}\left[\sum_{i=1}^{k} R_{i} \mid T=t\right]=\sum_{n=0}^{\infty} \mathrm{P}\left(N_{1}=n \mid T=t\right) n \mathrm{E}\left[Z^{(k)} \mid T=t\right]=\lambda p t g_{k}(t),
$$

where $g_{k}(t)=\mathrm{E}\left[Z^{(k)} \mid T=t\right], k=1,2, \ldots$, and $g_{0}(t)=0$. Hence, for $k=0,1, \ldots$,

$$
h_{k}(t)=\lambda(1-p) t+\lambda \operatorname{ptg}_{k}(t), \quad t>0 .
$$

For $k=1,2, \ldots$, to compute $\mathrm{E}\left[Z^{(k)} \mid T=t\right]$, let $V$ denote the excess lifetime of individual $A$ when our typical named descendant of $A, B$ say, is born, and let $T_{B}$ denote the natural lifetime of $B$. Then $(V \mid T=t) \sim U(0, t)$. Furthermore, if $T_{B} \geq V$ then, with probability $\pi, B$ can name its immediate offspring, while, with probability $1-\pi$, all $B$ 's immediate offspring are unnamed. So,

$$
\begin{gathered}
\left(Z^{(k)} \mid V=v, T_{B} \geq v\right) \stackrel{\mathrm{D}}{=}\left(R^{(k-1)} \mid T=v\right) \quad(B \text { can name }), \\
\mathrm{E}\left[Z^{(k)} \mid V=v, T_{B} \geq v\right]=\lambda v \quad(B \text { cannot name }),
\end{gathered}
$$

where ' $\stackrel{\text { D, }}{=}$ means 'equal in distribution to'. On the other hand, if $T_{B}<V$ then $B$ dies before $A$, so $B$ 's death is natural and $B$ names individuals in the same manner as an unnamed individual, i.e.

$$
\left(Z^{(k)} \mid V=v, T_{B}=t_{B}\right) \stackrel{\mathrm{D}}{=}\left(R^{(k-1)} \mid T=t_{B}\right), \quad t_{B}<v .
$$

Putting this all together yields

$$
\begin{aligned}
g_{k}(t) & =\int_{v=0}^{t} \frac{1}{t}\left\{\mathrm{P}\left(T_{B}>v\right)\left(\pi h_{k-1}(v)+\lambda(1-\pi) v\right)+\int_{u=0}^{v} \gamma \mathrm{e}^{-\gamma u} h_{k-1}(u) \mathrm{d} u\right\} \mathrm{d} v \\
& =\int_{v=0}^{t} \frac{1}{t}\left\{\mathrm{e}^{-\gamma v}\left(\pi h_{k-1}(v)+\lambda(1-\pi) v\right)+\int_{u=0}^{v} \gamma \mathrm{e}^{-\gamma u} h_{k-1}(u) \mathrm{d} u\right\} \mathrm{d} v,
\end{aligned}
$$


whence, using (4.2), $h_{0}(t)=\lambda(1-p) t$ and, for $k=1,2, \ldots$,

$$
\begin{aligned}
h_{k}(t)= & \lambda(1-p) t+\lambda p(1-\pi) \int_{0}^{t} \lambda v \mathrm{e}^{-\gamma v} \mathrm{~d} v+\lambda p \pi \int_{0}^{t} \mathrm{e}^{-\gamma v} h_{k-1}(v) \mathrm{d} v \\
& +\lambda p \int_{v=0}^{t} \int_{u=0}^{v} \gamma \mathrm{e}^{-\gamma u} h_{k-1}(u) \mathrm{d} u \mathrm{~d} v \\
= & \lambda(1-p) t+\frac{\lambda^{2} p(1-\pi)}{\gamma^{2}}\left\{1-\mathrm{e}^{-\gamma t}-\gamma t \mathrm{e}^{-\gamma t}\right\}+\lambda p \pi \int_{0}^{t} \mathrm{e}^{-\gamma v} h_{k-1}(v) \mathrm{d} v \\
& +\lambda p \int_{v=0}^{t} \int_{u=0}^{v} \gamma \mathrm{e}^{-\gamma u} h_{k-1}(u) \mathrm{d} u \mathrm{~d} v .
\end{aligned}
$$

For $\theta>0$, let $L_{k}(\theta)=\int_{0}^{\infty} \mathrm{e}^{-\theta t} h_{k}(t) \mathrm{d} t$ be the Laplace transform of $h_{k}(t)$. Hence, $L_{0}(\theta)=$ $\lambda(1-p) / \theta^{2}$ and taking the Laplace transform of (4.3) yields, for $k=1,2, \ldots$,

$$
L_{k}(\theta)=\frac{\lambda(1-p)}{\theta^{2}}+\frac{\lambda^{2} p(1-\pi)}{\theta(\theta+\gamma)^{2}}+\frac{\lambda p(\pi \theta+\gamma)}{\theta^{2}} L_{k-1}(\gamma+\theta) .
$$

Now, $R_{U}^{(k)}=\int_{0}^{\infty} \gamma \mathrm{e}^{-\gamma t} h_{k}(t) \mathrm{d} t=\gamma L_{k}(\gamma)$, and setting $\theta=j \gamma$ in (4.4) yields

$$
L_{k}(j \gamma)=\frac{\lambda(1-p)}{j^{2} \gamma^{2}}+\frac{\lambda^{2} p(1-\pi)}{j(j+1)^{2} \gamma^{3}}+\frac{\lambda p(\pi j+1)}{j^{2} \gamma} L_{k-1}((j+1) \gamma), \quad j=1,2, \ldots
$$

This gives

$$
L_{1}(j \gamma)=\frac{\lambda(1-p)}{j^{2} \gamma^{2}}+\frac{\lambda^{2} p(1-\pi)}{j(j+1)^{2} \gamma^{3}}+\frac{\lambda^{2} p(1-p)(\pi j+1)}{j^{2}(j+1)^{2} \gamma^{3}} .
$$

For $i, j=1,2, \ldots$, let $\hat{a}_{j, i}=\prod_{w=1}^{i-1}(\pi(j+w-1)+1)$. (In this paper all products are 1 if vacuous.)

Suppose that, for $k=1,2, \ldots, \kappa$ and $j=1,2, \ldots$,

$$
L_{k}(j \gamma)=\frac{\lambda(1-p)}{j^{2} \gamma^{2}}+\frac{\lambda p}{\gamma^{2}} \sum_{i=1}^{k} \frac{\lambda^{i} p^{i-1}((1-p \pi)(j+i-1)+1-p) \hat{a}_{j, i}}{\gamma^{i}[(i+j) !]^{2} /[(j-1) !]^{2}} .
$$

Then, using (4.5),

$$
\begin{aligned}
L_{\kappa+1}(j \gamma)= & \frac{\lambda(1-p)}{j^{2} \gamma^{2}}+\frac{\lambda^{2} p(1-\pi)}{j(j+1)^{2} \gamma^{3}}+\frac{\lambda p(\pi j+1)}{j^{2} \gamma} L_{\kappa}((j+1) \gamma) \\
= & \frac{\lambda(1-p)}{j^{2} \gamma^{2}}+\frac{\lambda^{2} p(1-\pi)}{j(j+1)^{2} \gamma^{3}}+\frac{\lambda^{2} p(1-p)(\pi j+1)}{j^{2}(j+1)^{2} \gamma^{3}} \\
& +\frac{\lambda p}{j^{2} \gamma^{2}} \sum_{i=1}^{\kappa} \frac{\lambda^{i+1} p^{i}((1-p \pi)(j+i)+1-p) \hat{a}_{j, i+1}}{\gamma^{i+1}[(i+j+1) !]^{2} /[j !]^{2}} \\
= & \frac{\lambda(1-p)}{j^{2} \gamma^{2}}+\frac{\lambda p}{\gamma^{2}} \sum_{i=1}^{\kappa+1} \frac{\lambda^{i} p^{i-1}((1-p \pi)(j+i-1)+1-p) \hat{a}_{j, i}}{\gamma^{i}[(i+j) !]^{2} /[(j-1) !]^{2}},
\end{aligned}
$$


so, by induction, (4.6) holds for all $k=1,2, \ldots$ Recall that $R_{U}^{(k)}=\gamma L_{k}(\gamma)$. Thus, letting $a_{i}=\hat{a}_{1, i}=\prod_{w=1}^{i-1}(\pi w+1), i=1,2, \ldots$, and setting $j=1$ in (4.6) yields

$$
R_{U}^{(k)}=\frac{\lambda(1-p)}{\gamma}+\frac{\lambda p}{\gamma} \sum_{i=1}^{k} \frac{\lambda^{i} p^{i-1}((1-p \pi) i+1-p) a_{i}}{\gamma^{i}[(i+1) !]^{2}} .
$$

Letting $k \rightarrow \infty$ in the above expression and using (4.1) gives

$$
R_{U}=\frac{\lambda(1-p)}{\gamma}+\frac{\lambda p}{\gamma} \sum_{i=1}^{\infty} \frac{\lambda^{i} p^{i-1}((1-p \pi) i+1-p) a_{i}}{\gamma^{i}[(i+1) !]^{2}} .
$$

Note that the expression for $R_{U}$ is convergent since

$$
p \sum_{i=1}^{\infty} \frac{\lambda^{i} p^{i-1}((1-p \pi) i+1-p) a_{i}}{\gamma^{i}[(i+1) !]^{2}}<\sum_{i=1}^{\infty} \frac{(\lambda p)^{i}(i+1) i !}{[(i+1) !]^{2} \gamma^{i}}<\sum_{i=1}^{\infty} \frac{(\lambda p)^{i}}{i ! \gamma^{i}}=\frac{\lambda}{\gamma}\left(\mathrm{e}^{\lambda p / \gamma}-1\right) .
$$

Letting $\pi=0$ in (4.7) yields

$$
R_{U}=1+\frac{\delta}{p} I_{-1}(2 \delta)-I_{0}(2 \delta)
$$

where $\delta=\sqrt{\lambda p / \gamma}$ and $I_{k}$ is the modified Bessel function of the first kind.

By conditioning on $A$ 's lifetime and the number of immediate offspring, we can obtain an integral equation involving $\mathrm{E}\left[s^{R} \mid T=t\right]$, details of which may be found in Chapter 3 of Knock (2011) for $\pi=0$ (but can be readily extended to more general $\pi$ ). However, it does not appear possible to solve this equation in order to obtain $H(s)$.

\subsection{A simplifying approximation: independence of sibling units}

The contact tracing considered here is such that individuals are named and traced at the end of their parent's lifetime, and, hence, sibling units codepend upon when their parent's lifetime ends, in other words upon the length of their parent's lifetime. Note that when the lifetime is constant, then the sibling units become independent, but what of the case where lifetimes are exponential? These intersibling dependencies make the model harder to analyse, but how much of an effect do they have?

Consider a typical type-1 individual in the MBDP. Their lifetime is the minimum of their natural lifetime and the excess lifetime of their parent when they are born (which have independent $\operatorname{Exp}(\gamma)$ distributions), and so the marginal distribution of a type- 1 individual's lifetime is $\operatorname{Exp}(2 \gamma)$. Hence, the marginal distribution of the excess lifetime of a type- 1 individual when they spawn a type-2 individual is $\operatorname{Exp}(2 \gamma)$, so the marginal distribution of a type-2 individual's lifetime is $\operatorname{Exp}(3 \gamma)$. Motivated by this marginal behaviour, we introduce an approximation to the MDBP, with independence of sibling units, as follows. Suppose that instead of being named at the end of their parent's lifetime, a typical type- $k$ individual (independently of their siblings and their natural lifetime) is named after a period of time from their birth, which has an $\operatorname{Exp}(k \gamma)$ distribution. The lifetime, $T_{k}$ say, of a type- $k$ individual would then be the minimum of this naming period and their natural lifetime (which is an $\operatorname{Exp}(\gamma)$ random variable), so in this approximation, $T_{k}$ has an $\operatorname{Exp}((k+1) \gamma)$ distribution. Furthermore, type- $k$ individuals would be traced with probability $k /(k+1)$, otherwise they would be untraced. Thus, a named individual would be asked to name their offspring, if any, with probability $(\pi k+1) /(k+1)$ 
(in which case their offspring are each named independently with probability $p$ ), otherwise all their offspring will be unnamed. Letting $M_{i j}$ be the mean number of type- $j$ offspring of a type- $i$ individual in this approximation,

$$
M_{k, 0}=\frac{\lambda(1-p)}{(k+1)^{2} \gamma}+\frac{k \lambda(1-\pi)}{(k+1)^{2} \gamma}+\frac{k \lambda(1-p) \pi}{(k+1)^{2} \gamma}, \quad M_{k, k+1}=\frac{\lambda p}{(k+1)^{2} \gamma}+\frac{k \lambda p \pi}{(k+1)^{2} \gamma} .
$$

Analogous to Section 2.2, we embed in this approximating multitype birth-death process a single (type-0) Galton-Watson branching process and let $\tilde{R}$ denote the offspring random variable of this GWP. Then

$$
\begin{aligned}
\mathrm{E}[\tilde{R}] & =M_{00}+M_{01}\left(M_{10}+M_{12}\left(M_{20}+M_{23}\left(M_{30}+\cdots\right)\right)\right) \\
& =M_{00}+\sum_{k=1}^{\infty}\left(\prod_{j=1}^{k} M_{j-1, j}\right) M_{k 0} \\
& =\frac{\lambda(1-p)}{\gamma}+\sum_{k=1}^{\infty} \frac{(\lambda p)^{k} a_{k}}{[k !]^{2} \gamma^{k}} \frac{\lambda(1-p+(1-p \pi) k)}{(k+1)^{2} \gamma} \\
& =\frac{\lambda(1-p)}{\gamma}+\frac{\lambda p}{\gamma} \sum_{k=1}^{\infty} \frac{\lambda^{k} p^{k-1}(1-p+(1-p \pi) k) a_{k}}{[(k+1) !]^{2} \gamma^{k}},
\end{aligned}
$$

which is the same as $R_{U}$.

Let $q_{k}, k=0,1,2, \ldots$, be the probability of extinction for this multitype birth-death process with one initial individual, who is of type $k$. Let $\phi_{k}(\theta)=\mathrm{E}\left[\mathrm{e}^{-\theta T_{k}}\right], \theta \geq 0$, be the moment generating function of $T_{k}$. Then, for $k=0,1, \ldots$, conditioning on $T_{k}$ and the numbers of type- 0 and type- $(k+1)$ individuals spawned by a type- $k$ individual having lifetime $T_{k}$ yields

$$
q_{k}=\frac{\pi k+1}{k+1} \phi_{k}\left(\lambda(1-p)\left(1-q_{0}\right)+\lambda p\left(1-q_{k+1}\right)\right)+\frac{(1-\pi) k}{k+1} \phi_{k}\left(\lambda\left(1-q_{0}\right)\right),
$$

whence, for $k=0,1, \ldots$,

$$
q_{k}=\frac{(\pi k+1) \gamma}{(k+1) \gamma+\lambda(1-p)\left(1-q_{0}\right)+\lambda p\left(1-q_{k+1}\right)}+\frac{(1-\pi) k \gamma}{(k+1) \gamma+\lambda\left(1-q_{0}\right)} .
$$

We consider two approximations to $q_{0}$. Let $j$ be a fixed positive integer. The first approximation assumes that type- $(j+1)$ individuals have no offspring, so $q_{k}^{(j, U)}, k=0,1, \ldots, j$, is obtained by setting $q_{j+1}=1$ in (4.8) and solving the resulting equations for $q_{0}, q_{1}, \ldots, q_{j}$. The second approximation assumes that type- $(j+1)$ individuals behave identically to type- $j$ individuals, so $q_{k}^{(j, L)}, k=0,1, \ldots, j$, is obtained by setting $q_{j+1}=q_{j}$ in (4.8) and solving the resulting equations for $q_{0}, q_{1}, \ldots, q_{j}$. In Appendix A we show that $q_{0}^{(j, L)} \uparrow q_{0}$ and $q_{0}^{(j, U)} \downarrow q_{0}$ as $j \rightarrow \infty$, thus providing a method for numerically evaluating $q_{0}$.

\section{Iterative tracing}

Setting $\pi=1$ in (3.1) and (4.7) yields

$$
R_{U}=\frac{1-p}{p}\left(\mathrm{e}^{\lambda p \mathrm{E}\left[T_{I}\right]}-1\right) .
$$

In this section we show that this holds for an arbitrary infectious period distribution. We do this by first assuming that $T_{I}$ has countable support and then assuming that it has an arbitrary distribution. 


\subsection{Infectious period with countable support}

Suppose that $T_{I}$ has countable support $\left\{t_{1}, t_{2}, t_{3}, \ldots\right\}$, where $0<t_{1}<t_{2}<t_{3}<\cdots$, such that $\mathrm{P}\left(T_{I}=t_{i}\right)=p_{i}>0, i=1,2, \ldots, \sum_{i=1}^{\infty} p_{i}=1$, and $\sum_{i=1}^{\infty} p_{i} t_{i}<\infty$.

To examine the offspring random variable of the GWP, we return to our typical unnamed individual $A$, having lifetime $T$ (so $T \stackrel{\mathrm{D}}{=} T_{I}$ ) in the MDBP. We need to find $R_{U}=\sum_{i=1}^{\infty} p_{i} h\left(t_{i}\right)$, where $h(t)=\mathrm{E}[R \mid T=t], 0 \leq t \leq \infty$. Although $T_{I}$ has countable support, we require $h(t)$ to have continuous support for the purpose of a renewal argument.

Recall that $R=R^{(0)}+\sum_{i=1}^{\infty} R_{i}$, where $R^{(0)}$ is the number of unnamed immediate descendants and $\sum_{i=1}^{\infty} R_{i}$ is the remaining number of offspring in the GWP. Now, $\left(R^{(0)} \mid T=t\right) \sim$ $\operatorname{Po}(\lambda(1-p) t)$, so $\mathrm{E}\left[R^{(0)} \mid T=t\right]=\lambda(1-p) t$.

Let $N_{1}$ denote the number of named immediate (i.e. type-1) offspring of $A$ and, for $i=$ $1,2, \ldots N_{1}$, let $Z_{i}$ be the total number of descendants from the $i$ th (arbitrarily ordered) such individual that contribute to $R$. Thus,

$$
\sum_{i=1}^{\infty} R_{i}=\sum_{i=1}^{N_{1}} Z_{i}
$$

Now $\left(N_{1} \mid T=t\right) \sim \operatorname{Po}(\lambda p t)$ and, conditional upon $N_{1}=k$ and $T=t$, the birth times of these $k$ individuals can be obtained by sampling $k$ independent $U(0, t)$ random variables, so the $Z_{i}$ are independent and identically distributed, with common distribution $Z$, say. Thus,

$$
\mathrm{E}\left[\sum_{i=1}^{\infty} R_{i} \mid T=t\right]=\sum_{k=0}^{\infty} \mathrm{P}\left(N_{1}=k \mid T=t\right) k \mathrm{E}[Z \mid T=t]=\lambda \operatorname{ptg}(t),
$$

where $g(t)=\mathrm{E}[Z \mid T=t]$, and it follows that

$$
h(t)=\lambda(1-p) t+\lambda \operatorname{ptg}(t) .
$$

To obtain $g(t)$, we return to our typical immediate named offspring of $A, B$, who is born $V$ time units before $A$ dies (i.e. $V \sim U(0, t))$ and has natural lifetime $T_{B}$. Let $t_{0}=0$. Suppose that $t_{i-1}<t \leq t_{i}, V=v$, and $j=\arg \max _{k}\left\{t_{k-1}: v \geq t_{k-1}\right\}$, i.e. $j$ is such that $v \in\left[t_{j-1}, t_{j}\right)$ for $j=1, \ldots, i-1$ or $v \in\left[t_{i-1}, t\right]$ for $j=i$. If $T_{B} \geq v$ then $B$ dies when $A$ dies and $B$ 's actual lifetime is $v$, while if $T_{B}<v, B$ 's actual lifetime is $T_{B}$. Hence,

$$
\left(Z \mid T_{B}=t_{k}\right) \stackrel{\mathrm{D}}{=} \begin{cases}\left(R \mid T=t_{k}\right), & k<j \\ (R \mid T=v), & k \geq j\end{cases}
$$

so

$$
g(t)=\sum_{j=1}^{i} \int_{v=t_{j-1}}^{\min \left(t_{j}, t\right)} \frac{1}{t}\left(\sum_{k=j}^{\infty} p_{k} h(v)+\sum_{k=1}^{j-1} p_{k} h\left(t_{k}\right)\right) \mathrm{d} v .
$$

Therefore, for $t_{i-1}<t \leq t_{i}$,

$$
h(t)=\lambda(1-p) t+\lambda p \sum_{j=1}^{i} \int_{v=t_{j-1}}^{\min \left(t_{j}, t\right)}\left(\sum_{k=j}^{\infty} p_{k} h(v)+\sum_{k=1}^{j-1} p_{k} h\left(t_{k}\right)\right) \mathrm{d} v,
$$


so

$$
h^{\prime}(t)=\lambda(1-p)+\lambda p \sum_{k=i}^{\infty} p_{k} h(t)+\lambda p \sum_{k=1}^{i-1} p_{k} h\left(t_{k}\right)
$$

which can be solved to give

$$
h(t) \sum_{k=i}^{\infty} p_{k}=\frac{A_{i}}{\lambda p} \exp \left\{\lambda p t \sum_{k=i}^{\infty} p_{k}\right\}-\frac{(1-p)}{p}-\sum_{k=1}^{i-1} p_{k} h\left(t_{k}\right)
$$

for some constant $A_{i}$.

Note that, for any $t \in[0, \infty), h(t+\Delta t)-h(t)$ is bounded above by the mean number of births in the untraced process (i.e. $p=0$ ) during $[t, t+\Delta t]$, since (a) not all births count towards $h$ and (b) the tracing has the effect of reducing lifetimes, and, therefore, the number of births. The mean number of births in the untraced process during $[t, t+\Delta t]$ tends to 0 as $\Delta t \downarrow 0$, so $h$ is continuous on $[0, \infty)$.

Since $h(t)$ is continuous, the values of the constants $A_{i}, i=1,2, \ldots$, can be determined by matching boundary values, and also by noting that $h(0)=0$. We thus obtain

$$
A_{i}=\lambda(1-p) \exp \left\{\lambda p \sum_{j=1}^{i-1} t_{j} p_{j}\right\}, \quad i=1,2, \ldots,
$$

so, for $t_{i-1}<t \leq t_{i}$

$$
h(t) \sum_{k=i}^{\infty} p_{k}=\frac{(1-p)}{p}\left(\exp \left\{\lambda p\left(\sum_{j=1}^{i-1} t_{j} p_{j}+t \sum_{k=i}^{\infty} p_{k}\right)\right\}-1\right)-\sum_{k=0}^{i-1} p_{k} h\left(t_{k}\right),
$$

whence

$$
\sum_{k=0}^{i} p_{k} h\left(t_{k}\right)+h\left(t_{i}\right) \sum_{k=i+1}^{\infty} p_{k}=\frac{(1-p)}{p}\left(\exp \left\{\lambda p\left(\sum_{j=1}^{i} t_{j} p_{j}+t_{i} \sum_{k=i+1}^{\infty} p_{k}\right)\right\}-1\right) .
$$

Now let $X$ be the random variable with mass function $\mathrm{P}\left(X=t_{k}\right)=p_{k}, k=1,2, \ldots$, and, for $i=1,2, \ldots$, let $X_{i}=\min \left(X, t_{i}\right)$. Then, we can rewrite (5.2) as

$$
\mathrm{E}\left[h\left(X_{i}\right)\right]=\frac{1-p}{p}\left(\mathrm{e}^{\lambda p \mathrm{E}\left[X_{i}\right]}-1\right) .
$$

Note that $X_{i} \uparrow X$ and $h\left(X_{i}\right) \uparrow h(X)$ almost surely as $i \rightarrow \infty$, and that $R_{U}=\mathrm{E}[h(X)]$. Thus, letting $i \rightarrow \infty$ in (5.3) and invoking the monotone convergence theorem yields (5.1).

\subsection{Arbitrarily distributed infectious period}

Suppose that $T_{I}$ has an arbitrary but specified distribution, satisfying $\mathrm{E}\left[T_{I}\right]<\infty$. For $h>0$, let $T_{h}^{-}=h\left\lfloor T_{I} / h\right\rfloor$ and $T_{h}^{+}=T_{h}^{-}+h$. (For $x \in \mathbb{R},\lfloor x\rfloor$ denotes the greatest integer not exceeding $x$.) Then $T_{h}^{-} \leq T_{I} \leq T_{h}^{+}$, so $\mathrm{E}\left[T_{h}^{-}\right] \leq \mathrm{E}\left[T_{I}\right] \leq \mathrm{E}\left[T_{h}^{+}\right]=\mathrm{E}\left[T_{h}^{-}\right]+h$ and a simple sandwiching argument shows that both $\mathrm{E}\left[T_{h}^{-}\right]$and $\mathrm{E}\left[T_{h}^{+}\right]$tend to $\mathrm{E}\left[T_{I}\right]$ as $h \downarrow 0$. 
We let $R_{h}^{-}$and $R_{h}^{+}$be the type-reproduction numbers when the natural lifetime has distribution $T_{h}^{-}$and $T_{h}^{+}$, respectively. By sampling shorter or longer natural lifetimes for $R_{h}^{-}$or $R_{h}^{+}$, respectively, it is clear that less or, respectively, more unnamed offspring are produced. Hence,

$$
R_{h}^{-} \leq R_{U} \leq R_{h}^{+}
$$

However, $T_{h}^{-}$and $T_{h}^{+}$both have countable support, so

$$
R_{h}^{-}=\frac{1-p}{p}\left(\mathrm{e}^{\lambda p \mathrm{E}\left[T_{h}^{-}\right]}-1\right) \quad \text { and } R_{h}^{+}=\frac{1-p}{p}\left(\mathrm{e}^{\lambda p \mathrm{E}\left[T_{h}^{+}\right]}-1\right) .
$$

Letting $h \downarrow 0$ and using the above limits for $\mathrm{E}\left[T_{h}^{-}\right]$and $\mathrm{E}\left[T_{h}^{+}\right]$yields (5.1).

\section{Numerical illustrations}

Throughout this section, we assume without loss of generality that $\mathrm{E}\left[T_{I}\right]=1$. The dependence of $\lambda_{\text {crit }}$ (the contact rate parameter value for which $R_{U}=1$ ) with the naming probability, $p$, is shown in Figure 2 for single-step tracing $(\pi=0)$, with exponential and constant infectious periods, and iterative tracing $(\pi=1)$. (Recall that, when $\pi=1, R_{U}$ depends on the infectious period distribution only through its mean, and, hence, so does $\lambda_{\text {crit }}$.) When the infectious period distribution is exponential, $\lambda_{\text {crit }}$ is higher (as we would expect since the median of an exponential distribution is below its mean, so infectious periods are generally shorter than in the constant case), and the range of values of $\lambda$, for which the exponential case is subcritical while the constant case is supercritical, increases with $p$. As $p \rightarrow 1, \lambda_{\text {crit }} \rightarrow \infty$ in the iterative case, since everyone is being named and there are no unnamed offspring. We can see that the effectiveness of iterative tracing over single-step tracing as measured by $\lambda_{\text {crit }}$ may be minor for small $p$, but increases greatly as $p$ does.

While we do not have an analytical expression from which to obtain the extinction probability $p_{\mathrm{e}}$ in supercritical exponentially distributed infectious period cases, we can estimate it by simulating the offspring random variable $R$ in the GWP. In Figure 3, we examine how $p_{\mathrm{e}}$ varies with $\lambda$, using the analytical values in the constant infectious period cases and estimated values
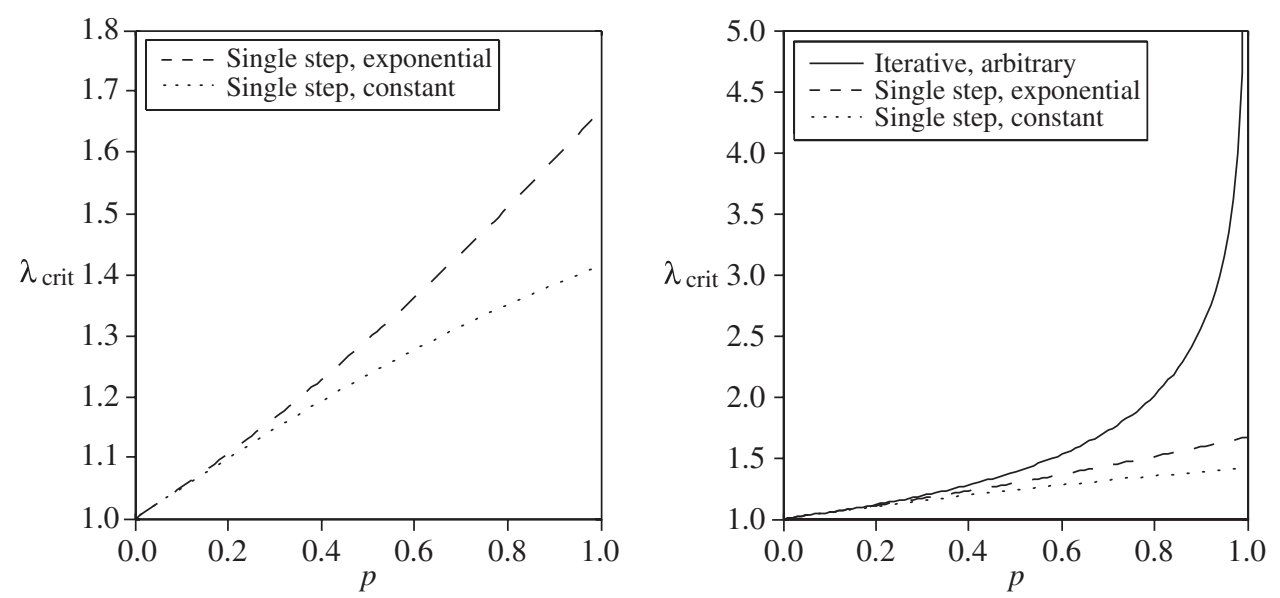

FIGURE 2: $\lambda_{\text {crit }}$ varying with $p$ for single-step tracing (exponential and constant infectious periods) and iterative tracing (arbitrary infectious period) when $\mathrm{E}\left[T_{I}\right]=1$. The plot on the left omits the iterative case to highlight the difference between the single-step cases. 

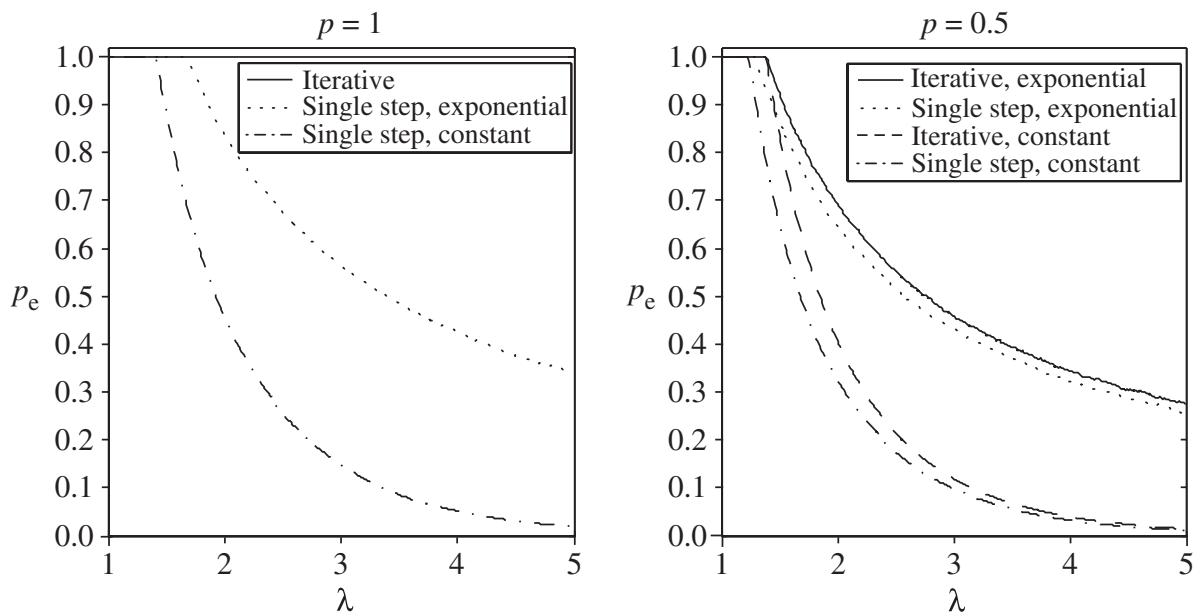

FIGURE 3: $p_{\mathrm{e}}$ varying with $\lambda$ when $\mathrm{E}\left[T_{I}\right]=1$.

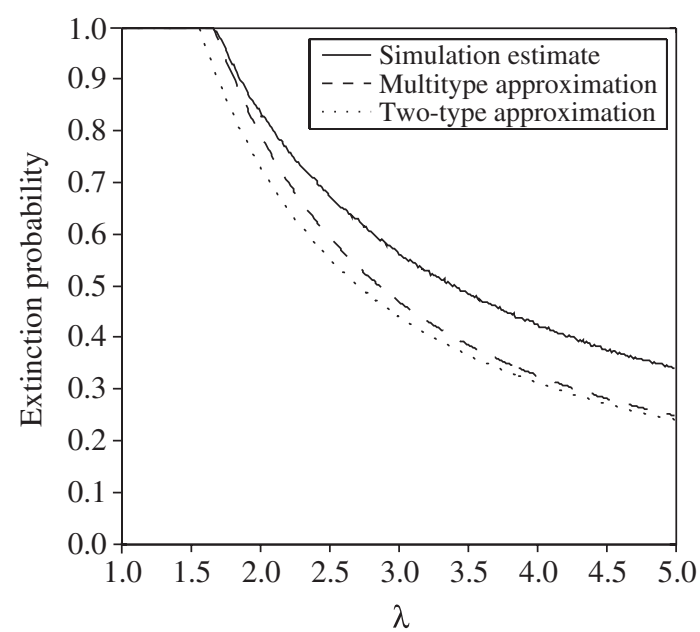

FIGURE 4: Comparing $\lambda_{\text {crit }}$ and the extinction probabilities of the two-type and multitype approximations with those of the full model, with $T_{I} \sim \operatorname{Exp}(1)$ and $\pi=0$. Extinction probabilities are examined when

$$
p=1 \text {. }
$$

from simulations in the exponential infectious period cases (any 'wiggles' exhibited in the curve arise from randomness, we would expect the true curve to be smooth). These estimates are obtained by simulating $R 100000$ times to obtain an empirical distribution for it; our estimate is then given as the solution of $\bar{H}(s)=s$, in $(0,1)$, where $\bar{H}(s)$ is the empirical probability generating function of $R$. There is a clear difference between the iterative and single-step cases when $p=1$ (and $p_{\mathrm{e}}=1$ in the iterative case), but, except for fairly small $\lambda$ in the constant infectious period case, this becomes very small when $p=0.5$. However, both when $p=0.5$ and when $p=1$, the difference between the exponential and constant infectious period cases is pronounced.

In Figure 4 we compare $\lambda_{\text {crit }}$ and the extinction probabilities of the true tracing model with those of the multitype approximation discussed in Section 4.1 and a cruder, two-type 
approximation given by $q_{0}^{(1, L)}$ (wherein type-2 individuals behave identically to type- 1 individuals) when $T_{I} \sim \operatorname{Exp}(1)$ and $\pi=0$. For smaller subcritical values of $\lambda$, the multitype approximation is close to giving the true extinction probability (again simulations have been used); however, it is not significantly better than the two-type approximation for larger $\lambda$. This is perhaps because, as $\lambda$ gets larger, the offspring random variable in the GWP, $R$, will generally be small or large, and the nature of this dichotomy will be dominated by the earlier levels in a naming tree, so the reduction of lifetimes further down the naming tree will have less of an impact. Generally, the approximations are fairly reasonable, though, for larger $\lambda$, the extinction probability is underestimated by about 0.1 . Another two-type approximation, given by $q_{0}^{(1, U)}$, can be considered, though it is generally even cruder, particularly for smaller subcritical values of $\lambda$.

With the two-type approximation, named individuals have the same lifetime distribution, no matter how far removed they are from an unnamed ancestor, so this is the likely reason that it overestimates the spread of the true model, in which lifetimes are reduced as you go down the naming tree. We also see that the multitype approximation overestimates the spread of the true model, so it seems that the intersibling dependencies have an effect of reducing spread. This may be because if an individual has a short lifetime then their siblings are more likely to, and, furthermore, all their named offspring are then more likely to have much shorter lifetimes, and so on.

\section{Appendix A}

In the appendix we prove the properties of $\left(q_{0}^{(j, L)}\right)$ and $\left(q_{0}^{(j, U)}\right)$ stated at the end of Section 4.1. Note that $q_{0}$ is the smallest root in $[0,1]$ of $f_{\tilde{R}}(s)=s$, where $f_{\tilde{R}}(s)=\mathrm{E}\left[s^{\tilde{R}}\right]$. For $j=1,2, \ldots$, let $\tilde{R}_{j}^{U}$ be the offspring random variable of the corresponding embedded type- 0 GWP when type- $(j+1)$ individuals have no offspring and let $\tilde{R}_{j}^{L}$ be the offspring random variable of the corresponding embedded type-0 GWP when type- $(j+1)$ individuals behave identically to type- $j$ individuals. Then $q_{0}^{(j, L)}$ and $q_{0}^{(j, U)}$ are given by the smallest roots in $[0,1]$ of $f_{j}^{L}(s)=s$ and $f_{j}^{U}(s)=s$, respectively, where $f_{j}^{L}(s)=\mathrm{E}\left[s^{\tilde{R}_{j}^{L}}\right]$ and $f_{j}^{U}(s)=\mathrm{E}\left[s^{\tilde{R}_{j}^{U}}\right]$.

Note that $R_{U}=\mathrm{E}[\tilde{R}]$, and recall that $R_{U}<\infty$, so $\mathrm{P}(\tilde{R}<\infty)=1$. It is straightforward to define $\tilde{R}, \tilde{R}_{1}^{U}, \tilde{R}_{2}^{U}, \ldots$ and $\tilde{R}_{1}^{L}, \tilde{R}_{2}^{L}, \ldots$ on a common probability space so that, almost surely, $\tilde{R}_{j}^{U} \uparrow \tilde{R}$ and $\tilde{R}_{j}^{L} \rightarrow \tilde{R}$ as $j \rightarrow \infty$. Thus,

$$
\tilde{R}_{j}^{U} \stackrel{\mathrm{D}}{\rightarrow} \tilde{R} \quad \text { and } \quad \tilde{R}_{j}^{L} \stackrel{\mathrm{D}}{\rightarrow} \tilde{R} \quad \text { as } j \rightarrow \infty
$$

where ' $\stackrel{\mathrm{D}}{\rightarrow}$ ' denotes convergence in distribution, and it follows using Lemma 4.1 of Britton et al. (2007) that $q_{0}^{(j, U)} \rightarrow q_{0}$ and $q_{0}^{(j, L)} \rightarrow q_{0}$ as $j \rightarrow \infty$. The sequence $\left(q_{0}^{(j, U)}\right)$ is clearly decreasing, since $\left(\tilde{R}_{j}^{U}\right)$ is stochastically increasing, so to complete the proof, it is sufficient to show that, for $j=1,2, \ldots, f_{j}^{L}(s) \leq f_{j+1}^{L}(s), 0 \leq s \leq 1$.

Consider a realisation of the approximating multitype birth-death process with a single ancestor, having type 0 , viewed on a generation basis. For $j=1,2, \ldots$, let $Y_{j}$ be the number of type- $j$ individuals in generation $j$ (these individuals have no type- 0 individual between them and the ancestor in the family tree), and let $W_{j}$ be the total number of type- 0 individuals in generations $1,2, \ldots, j$ who have no type- 0 individual between them and the ancestor in the family tree. Also, let $X_{j}$ be the total number of type- 0 progeny in a two-type ( 0 and $j$ ) process, with one ancestor who is of type- $j$, in which only type- $j$ individuals produce offspring, and a typical type- $j$ individual has lifetime $T_{j} \sim \operatorname{Exp}((j+1) \gamma)$ and has offspring at rate $\lambda$. Moreover, with probability $(1-\pi) j /(j+1)$, all her offspring have type 0 , otherwise the type of each of 
her offspring is chosen independently, with $\mathrm{P}($ type $j)=p=1-\mathrm{P}($ type 0$)$. Observe that, for $j=1,2, \ldots$,

$$
\tilde{R}_{j}^{L} \stackrel{\mathrm{D}}{=} W_{j+1}+\sum_{i=1}^{Y_{j+1}} X_{j}^{(i)} \quad \text { and } \quad \tilde{R}_{j+1}^{L} \stackrel{\mathrm{D}}{=} W_{j+1}+\sum_{i=1}^{Y_{j+1}} X_{j+1}^{(i)},
$$

where, for example, $X_{j}^{(1)}, X_{j}^{(2)}, \ldots$ are independent and identically distributed copies of $X_{j}$. The decompositions in (A.1) imply that, for $0 \leq s \leq 1, f_{j}^{L}(s) \leq f_{j+1}^{L}(s)$ if and only if $f_{j}(s) \leq f_{j+1}(s)$, where $f_{j}(s)=\mathrm{E}\left[s^{X_{j}}\right]$.

Suppose without loss of generality that $\gamma=1$. Conditioning on $T_{j}$ yields

$$
f_{j}(s)=\frac{(1-\pi) j}{j+1+\lambda(1-s)}+\frac{1+\pi j}{j+1+\lambda p\left(1-f_{j}(s)\right)+\lambda(1-p)(1-s)},
$$

so $f_{j}(s)$ satisfies the quadratic equation $g_{j}(x)=0$, where

$$
\begin{gathered}
g_{j}(x)=\lambda p x^{2}-b_{j} x+c_{j}, \\
b_{j}=j+1+\lambda(1-s)+\lambda p s+\frac{\lambda p j(1-\pi)}{j+1+\lambda(1-s)},
\end{gathered}
$$

and

$$
c_{j}=j+1+\frac{\lambda p j(1-\pi) s}{j+1+\lambda(1-s)} .
$$

Now, $g_{j}(1)=-\lambda(1-p)(1-s)-\lambda p j(1-\pi)(1-s) /(j+1+\lambda(1-s))$, so, for $s \in[0,1)$, $g_{j}(1)<0$ unless $p=\pi=1$. If $p=\pi=1$, all individuals are named and the entire embedding approach breaks down. Thus, for all cases of interest, $f_{j}(s)$ is the smallest solution of $g_{j}(x)=0$. Fix $j \geq 2$. Simple algebra yields

$$
g_{j}(x)-g_{j-1}(x) \geq 0 \quad \Longleftrightarrow \quad x \leq x_{0}=\frac{1+s d_{j}}{1+d_{j}},
$$

where

$$
d_{j}=\frac{\lambda p(1-\pi)(1+\lambda(1-s))}{(j+1+\lambda(1-s))(j+\lambda(1-s))} .
$$

Thus, since $g_{j}(0)>g_{j-1}(0)$ and $f_{j}(s)$ is the smallest solution of $g_{j}(x)=0$, a sufficient condition for $f_{j}(s) \geq f_{j+1}(s)$ is $g_{j}\left(x_{0}\right) \leq 0$.

Lengthy algebra, noting that $1-x_{0}=(1-s) d_{j} /\left(1+d_{j}\right), x_{0}-s=(1-s) /\left(1+d_{j}\right)$, and $\lambda p(1-\pi) /(j+1+\lambda(1-s))=((j+\lambda(1-s)) /(1+\lambda(1-s))) d_{j}$, and setting $r_{j}(s)=$ $1-\lambda s-j(j-1) /(1+\lambda(1-s))$, yields

$$
\frac{\left(1+d_{j}\right)^{2} g_{j}\left(x_{0}\right)}{1-s}=d_{j}\left(1+d_{j}\right) r_{j}(s)-\lambda\left(1-p+d_{j}(1-p s)\right) .
$$

Hence, $g_{j}\left(x_{0}\right) \leq 0$ if $r_{j}(s) \leq 0$, so we now suppose that $r_{j}(s)>0$. Let

$$
h_{j}(x)=x^{2} r_{j}(s)+x\left(r_{j}(s)-\lambda(1-p s)\right)-\lambda(1-p) .
$$


Then $g_{j}\left(x_{0}\right) \leq 0$ if and only if $h_{j}\left(d_{j}\right) \leq 0$. Suppose that $p<1$. Then, there exists $x_{j}^{*}>0$ such that $h_{j}(x) \leq 0$ for $x \in\left[0, x_{j}^{*}\right]$ and $h_{j}(x)>0$ for $x \in\left(x_{j}^{*}, \infty\right)$. Note that $x_{j}^{*}$ is independent of $\pi$ and that $d_{j}$ decreases with $\pi$. Thus, if $g_{j}\left(x_{0}\right) \leq 0$ when $\pi=0$ then $g_{j}\left(x_{0}\right) \leq 0$ for all $\pi \in[0,1]$. The same conclusion holds when $p=1$. Hence, it is sufficient to consider the case $\pi=0$.

Let $\pi=0$ and write $d_{j}=p e_{j}$. Then $g_{j}\left(x_{0}\right) \leq 0$ if and only if $H_{j}(p) \leq 0$, where

$$
H_{j}(p)=p^{2}\left(e_{j}^{2} r_{j}(s)+\lambda e_{j} s\right)+p\left(e_{j}\left(r_{j}(s)-\lambda\right)+\lambda\right)-\lambda .
$$

Recall that $r_{j}(s)>0$. Then $H_{j}(1) \leq 0$ implies that $H_{j}(p) \leq 0$ for all $p \in[0,1]$, so we need consider only $p=1$. Thus, setting $p=1$ in (A.2), we need to show that $\mathrm{e}_{j}^{2} r_{j}(s)+e_{j}\left(r_{j}(s)-\right.$ $\lambda(1-s)) \leq 0$, or, equivalently, since $e_{j}>0$, that $e_{j} \leq y_{j}$, where $y_{j}=\lambda(1-s) / r_{j}(s)-1$. Now, $e_{j}$ decreases with $j$ and, provided that $r_{j}(s)>0, y_{j}$ increases with $j$, so it is sufficient to consider $j=2$. Thus, noting that $e_{j}=d_{j}$ as $p=1$, we need to show that

$$
\frac{\lambda(1+\lambda(1-s))}{(2+\lambda(1-s))(3+\lambda(1-s))} \leq \frac{\lambda(1-s)}{1-\lambda s-2 /(1+\lambda(1-s))}-1 .
$$

Let $z=1+\lambda(1-s)$, so $z \in[1,1+\lambda]$ and $z-\lambda-2 z^{-1}>0$. Elementary algebra then shows that (A.3) is equivalent to

$$
z^{3} \leq\left(\lambda^{2}+3 \lambda-1\right) z^{2}+4(1+\lambda) z+4
$$

which clearly holds as $z^{3} \leq(1+\lambda) z^{2}$ and $\lambda>1$, since otherwise the birth-death process is necessarily not supercritical.

\section{Acknowledgement}

Edward Knock was supported by an EPSRC Doctoral Training grant.

\section{References}

AthreYA, K. B. AND KARLIN, S. (1971). On branching processes with random environments. I. Extinction probabilities. Ann. Math. Statist. 42, 1499-1520.

Ball, F. G. (1983). The threshold behaviour of epidemic models. J. Appl. Prob. 20, 227-241.

Ball, F. ANd Donnelly, P. (1995). Strong approximations for epidemic models. Stoch. Process. Appl. 55, 1-21.

Ball, F. G., KNOCK, E. S. ANd O’Neill, P. D. (2008). Control of emerging infectious diseases using responsive imperfect vaccination and isolation. Math. Biosci. 216, 100-113.

Ball, F., O’Neill, P. D. AND Pike, J. (2007). Stochastic epidemic models in structured populations featuring dynamic vaccination and isolation. J. Appl. Prob. 44, 571-585.

Becker, N. G., Glass, K., Li, Z. AND Aldis, G. K. (2005). Controlling emerging infectious diseases like SARS. Math. Biosci. 193, 205-221.

Britton, T., Janson, S. And Martin-LöF, A. (2007). Graphs with specified degree distributions, simple epidemics, and local vaccination strategies. Adv. Appl. Prob. 39, 922-948.

Haccou, P., Jagers, P. and Vatutin, V. A. (2005). Branching processes: Variation, Growth, and Extinction of Populations. Cambridge University Press.

Heesterbeek, J. A. P. and Roberts, M. G. (2007). The type-reproduction number $T$ in models for infectious disease control. Math. Biosci. 206, 3-10.

Kaplan, E. H., Craft, D. L. AND Wein, L. M. (2002). Emergency response to a smallpox attack: the case for mass vaccination. Proc. Nat. Acad. Sci. USA 99, 10935-10940.

KLEBANER, F. C. (1989). Linear growth in near-critical population-size-dependent multitype Galton-Watson processes. J. Appl. Prob. 26, 431-445.

Klinkenberg, D., Fraser, C. And Heesterbeek, H. (2006). The effectiveness of contact tracing in emerging epidemics. PLOS ONE 1, e13. 
KNOCK, E. S. (2011). Stochastic epidemic models for emerging diseases incorporating household structure and contact tracing. Doctoral Thesis, University of Nottingham. Available at http://etheses.nottingham.ac.uk/2046.

Lambert, A. (2010). The contour of splitting trees is a Lévy process. Ann. Prob. 38, 348-395.

Müller, J., Kretzschmar, M. And Dietz, K. (2000). Contact tracing in stochastic and deterministic epidemic models. Math. Biosci. 164, 39-64.

Olofsson, P. (1996). Branching processes with local dependencies. Ann. Appl. Prob. 6, 238-268.

Shaban, N., Andersson, M., Svensson, Å. and Britton, T. (2008). Networks, epidemics and vaccination through contact tracing. Math. Biosci. 216, 1-8. 